PROCEEDINGS OF THE

AMERICAN MATHEMATICAL SOCIETY

Volume 127, Number 8, Pages 2449-2458

S 0002-9939(99)04783-8

Article electronically published on April 8, 1999

\title{
ON LINEARLY LINDELÖF AND STRONGLY DISCRETELY LINDELÖF SPACES
}

\author{
A. V. ARHANGEL'SKII AND R. Z. BUZYAKOVA \\ (Communicated by Alan Dow)
}

\begin{abstract}
We prove that the cardinality of every first countable linearly Lindelöf Tychonoff space does not exceed $2^{\omega}$, and every strongly discretely Lindelöf Tychonoff space of countable tightness is Lindelöf.
\end{abstract}

\section{$\S 1$. INTRODUCTION}

It is well known that a space $X$ is compact if and only if every infinite set in $X$ has a point of complete accumulation [1]. So it is rather amusing to notice that the following condition (introduced in [1]):

(CAP) every uncountable subset $A$ of $X$ of regular cardinality has a point of complete accumulation in $X$,

does not characterize Lindelöf spaces, though it is not difficult to show that all Lindelöf spaces satisfy (CAP). Probably, the first example of a space of this kind was constructed by A. S. Mischenko [7]. The spaces satisfying (CAP) were later renamed into linearly Lindelöf, or chain-Lindelöf, spaces since the condition (CAP) turned out to be equivalent to the following requirement: every open covering $\gamma$ of $X$ which is a chain (that is, for any two elements of $\gamma$, one is a subset of the other one) contains a countable subcovering of $X$.

It is natural to consider the next two general questions:

Question 1. Which additional conditions force a linearly Lindelöf space to be Lindelöf?

Question 2. Which theorems on Lindelöf spaces can be extended to linearly Lindelöf spaces?

In this paper we formulate several concrete questions of the first and second type. We also make a positive contribution in both directions. In particular, we prove that the cardinality of a first countable linearly Lindelöf space does not exceed $2^{\omega}$ (Theorem 3.1.), which is a generalization of the main theorem in [2]. All spaces considered are assumed to be $T_{1}$. Note, that it is a famous open problem whether there exists a normal linearly Lindelöf space which is not Lindelöf (see [8]). The importance of this problem is partly explained by the fact that a normal linearly

Received by the editors June 27, 1997 and, in revised form, October 27, 1997.

1991 Mathematics Subject Classification. Primary 54A25; Secondary 54A35.

Key words and phrases. Lindelöf space, linearly Lindelöf space, free sequence, $G_{2} \omega$-set, first countability, complete accumulation point.

The first author was partially supported by NSF-grant DMS-9312363. 
Lindelöf non-Lindelöf space would be a particularly nice Dowker space because of the following well known fact related to Question 1: every linearly Lindelöf countably paracompact space is Lindelöf (see [7] and [8]).

\section{§2. Some technical RESUlts}

In what follows, $\tau$ is an infinite cardinal number, which we also interpret as the first ordinal number of cardinality $\tau$. We call a subset $A$ of a space $X$ a $G_{\tau^{-}}$set in $X$, or a subset of the type $G_{\tau}$, if there is a family $\gamma$ of open subsets of $X$ such that the cardinality of $\gamma$ is not greater than $\tau$ and $A$ is the intersection of $\gamma$. We denote by $C h(X)$ the smallest infinite cardinal number $\tau$ such that $X$ is of the type $G_{\tau}$ in $\beta(X)$ (or, which is equivalent, $X$ is of the type $G_{\tau}$ in some other Hausdorff compactification of $X)$.

A point $x$ of a space $X$ is called $G_{\tau}$-separated from a subset $Y$ of $X$, if there is a closed $G_{\tau}$-set $P$ in $X$ such that $x \in P$ and the sets $Y$ and $P$ are disjoint.

We recall also the notion of a free sequence introduced in [2]. Let $\kappa$ be an ordinal number. A $\kappa$-long free sequence in a space $X$ is a transfinite sequence $S=\left\{x_{\alpha}: \alpha<\kappa\right\}$ of elements of $X$ such that for every $\alpha<\kappa$ the closures in $X$ of the sets

$$
L_{S}(\alpha)=\left\{x_{\beta}: \beta<\alpha\right\} \quad \text { and } \quad R_{S}(\alpha)=\left\{x_{\beta}: \alpha \leq \beta<\kappa\right\}
$$

are disjoint.

First, we will need the next simple result belonging to the folklore, which is proved by an easy, straightforward argument.

Lemma 2.1. Let $X$ be a Hausdorff sequential space the cardinality of which is greater than $2^{\omega}$, and let $\tau$ be the first cardinal number which is greater than $2^{\omega}$. Then there is a closed subspace $Y$ of $X$ such that the cardinality of $Y$ is $\tau$.

Proof. Clearly, there is an increasing chain $\eta=\left\{M_{\alpha}: \alpha<\tau\right\}$ of subsets of $X$ such that $\left|M_{\alpha}\right| \leq 2^{\omega}$, for each $\alpha<\tau$, and $|\bigcup \eta|=\tau$. Put $F_{\alpha}=\overline{M_{\alpha}}$ and $Y=\bigcup\left\{F_{\alpha}\right.$ : $\alpha<\tau\}$. Then $\left|F_{\alpha}\right| \leq 2^{\omega}$, since $X$ is Hausdorff and sequential (see [2], [3]), and, therefore, $|Y| \leq \tau$. It follows that $|Y|=\tau$, since $\tau=|\bigcup \eta| \leq|Y|$. Besides, $Y$ is closed in $X$, since the tightness of $X$ is countable, $\tau$ is a not countably cofinal, and $\left\{F_{\alpha}: \alpha<\tau\right\}$ is a $\tau$-long increasing sequence of closed sets. Thus, $Y$ is a subspace of $X$, which we were looking for.

The next result has a well known prototype (see [3]); still, there is something new in it.

Lemma 2.2. Let $Z$ be a regular space, $A$ a subset of $Z$, and $z$ a point of $Z$. Then at least one of the following three conditions is satisfied:

a) There is a countable discrete, in itself (in fact, a free sequence in $A$ ), subset $B$ of $A$ such that $z$ is not $G_{\omega}$-separated from the closure of $B$ in $A$;

b) $z$ is $G_{\omega}$-separated from $A$;

c) There is an $\omega_{1}$-long free sequence in the space $A$.

Proof. Assume that neither condition a), nor condition b) is satisfied. Then, since $Z$ is regular, $z \notin A$, but $z \in \bar{A}$. In particular, $A$ is not empty.

We will construct an $\omega_{1}$-long free sequence in $Z$ contained in $A$ by transfinite recursion as follows. Simultaneously, we will be building up a decreasing transfinite sequence of closed $G_{\omega}$-subsets of $Z$ containing $z$. 
Let $x_{0}$ be any point of $A$ and $F_{0}=Z$. Let $\alpha \leq \omega_{1}$. Assume that for each $\beta<\alpha$ a point $x_{\beta} \in A$ and a closed $G_{\omega}$-subset $F_{\beta}$ in $Z$, containing the point $z$, are already chosen in such a way that the next conditions are satisfied:

d) $x_{\beta} \in F_{\beta}$;

e) $F_{\beta}$ does not intersect the closure in $A$ of the set $S_{\beta}=\left\{x_{\kappa}: \kappa<\beta\right\}$;

f) $F_{\beta} \subset F_{\kappa}$, for each $\kappa<\beta$.

From conditions d), e), and f) it follows that the set $M_{\beta}=\left\{x_{\kappa}: \beta \leq \kappa<\alpha\right\}$ is contained in the set $F_{\beta}$. Therefore the closure of $M_{\beta}$ in $Z$ is contained in $F_{\beta}$. On the other hand, the closure of $S_{\beta}$ in $A$ does not intersect $F_{\beta}$ by condition e). It follows that $\xi=\left\{x_{\beta}: \beta<\alpha\right\}$ is a free sequence in $A$. If $\alpha=\omega_{1}$, we stop the construction.

Now let $\alpha<\omega_{1}$. Then $S_{\alpha}$ is a discrete countable subset of $A$; since condition a) is not satisfied, $z$ is $G_{\omega}$-separated from the closure of $S_{\alpha}$ in $A$. Therefore, there exists a closed $G_{\omega}$-set $P_{\alpha}$ in $Z$ such that $z \in P_{\alpha}$ and

g) $P_{\alpha} \cap \overline{S_{\alpha}}=\emptyset$.

Now put

$$
F_{\alpha}=P_{\alpha} \cap\left(\bigcap\left\{F_{\beta}: \beta<\alpha\right\}\right) .
$$

Clearly, $F_{\alpha}$ is a closed $G_{\omega}$-subset of $Z$ containing $z$. Therefore, since condition b) is not satisfied, $A \cap F_{\alpha} \neq \emptyset$. Let $x_{\alpha}$ be any point of $A \cap F_{\alpha}$. Conditions d), e) and $\mathrm{f}$ ) are now satisfied for each $\beta \leq \alpha$. The definition of transfinite sequences $\left\{x_{\alpha}: \alpha<\omega_{1}\right\}$ and $\left\{F_{\alpha}: \alpha<\omega_{1}\right\}$ is complete. We have already shown above that $\left\{x_{\alpha}: \alpha<\omega_{1}\right\}$ is a free sequence in $A$. Thus, condition c) is satisfied, and the proof of Lemma 2.2 is complete.

If every free sequence in $X$ is countable, we write $F(X) \leq \omega$.

We will need the following special case of Lemma 2.2:

Lemma 2.3. Let $Y$ be a subspace of $X$ and $x \in X$. Assume also that $F(Y) \leq \omega$. Then either

a) There is a countable discrete subset $A$ of $Y$ such that $x$ is in the closure of $A$, or

b) There is a closed $G_{\omega}$-set $P$ in $X$ such that $x \in P$ and $P \cap Y=\emptyset$.

Lemma 2.4. Let $X$ be a linearly Lindelöf space and $\mathcal{P}$ a family of closed subsets of $X$, satisfying the next two conditions:

a) $\cap \mathcal{P}=\emptyset$, and

b) for every closed subset $P$ of $X$ there is $F \in \mathcal{P}$ such that either $F \subset P$ or $F \cap P=\emptyset$.

Then there is a countable subfamily $\xi$ of $\mathcal{P}$ such that $\bigcap \xi=\emptyset$.

Proof. Let $\tau$ be the smallest cardinal number such that there is a subfamily $\gamma$ of $\mathcal{P}$ satisfying the conditions: $\bigcap \gamma=\emptyset$ and $|\gamma|=\tau$. If $\tau$ is countable, we are done. Assume now that $\tau$ is uncountable. Then the cofinality of $\tau$ is $\omega$, since $X$ is linearly Lindelöf. Therefore, $\gamma=\bigcup\left\{\gamma_{n}: n \in \omega\right\}$, where $\left|\gamma_{n}\right|<\tau$, for each $n \in \omega$. Then $\bigcap\left(\gamma_{n}\right)$ is a closed subset $P_{n}$ of $X$ and, in virtue of condition b), there is $F_{n} \in \mathcal{P}$ such that either $F_{n} \subset P_{n}$ or $F_{n} \cap P_{n}=\emptyset$. Now, the second case is impossible since when we add $F_{n}$ to the family $\gamma_{n}$ (as an element), we obtain a subfamily $\eta_{n}$ of $\mathcal{P}$ such that $\left|\eta_{n}\right|<\tau$ and, therefore, $\bigcap\left(\eta_{n}\right) \neq \emptyset$. It follows that $F_{n} \subset P_{n}$, for each $n \in \omega$. 
Clearly, $\bigcap\left\{P_{n}: n \in \omega\right\}=\bigcap \gamma=\emptyset$. Therefore, $\bigcap\left\{F_{n}: n \in \omega\right\}=\emptyset$, that is, $\xi=\left\{F_{n}: n \in \omega\right\}$ is a countable subfamily of $\gamma$ that we were looking for.

Proposition 2.5. Let $X$ be a regular space, $Y$ a linearly Lindelöf subspace of $X$ such that $F(Y) \leq \omega$, and $x \in(X \backslash Y)$. Also let $\mathcal{P}$ be a family of closed subsets of $Y$ satisfying the next two conditions:

a) every closed separable subspace $F$ of the space $Y$ such that $x$ is in the closure of $F$ in the space $X$ belongs to $\mathcal{P}$, and

b) for every closed $G_{\omega}$-subset $B$ of the space $X$ such that $x \in B$, the set $Y \cap B$ belongs to $\mathcal{P}$.

Then there is a countable subfamily $\xi$ of $\mathcal{P}$ such that $\bigcap \xi=\emptyset$.

Proof. Let $P$ be a subset of $Y$ closed in $Y$. Then $F(P) \leq \omega$ and, in virtue of Lemma 2.3 , either there exists a closed $G_{\omega}$-set $B$ in $X$ such that $x \in B$ and $P \cap B=\emptyset$ or, there exists a separable subspace $F$ of $P$ closed in $P$ such that $x$ is in the closure of $F$ in $X$. In the first case, $B \cap Y$ is an element of $\mathcal{P}$ disjoint from $P$; in the second case, $F$ is an element of $\mathcal{P}$ contained in $P$. Since $x$ is not in $Y$, and $Y$ is regular, it is clear that the intersection of $\mathcal{P}$ is empty. Therefore, $Y$ and $\mathcal{P}$ satisfy all conditions in Lemma 2.4 and, in virtue of the Lemma, there is a countable subfamily $\xi$ of $\mathcal{P}$ such that $\bigcap \xi=\emptyset$.

Proposition 2.6. Let $X$ be a linearly Lindelöf Tychonoff space of countable tightness such that $|X| \leq 2^{\omega}$ and $w(X) \leq 2^{\omega}$. Then $C h(X) \leq 2^{\omega}$.

Proof. Let $Z=b(X)$ be a Hausdorff compactification of $X$ such that $w(Z) \leq 2^{\omega}$ and let $\mathcal{S}$ be the family of all closed subsets $B$ of $Z$ such that $B \cap X$ is separable. Since $|X| \leq 2^{\omega}$, the cardinality of $\mathcal{S}$ is not greater than $2^{\omega}$. Let $\mathcal{G}$ be the family of all closed $G_{\omega}$-subsets of $Z$. Since $Z$ is compact and $w(Z) \leq 2^{\omega}$, we have $|\mathcal{G}| \leq 2^{\omega}$. Therefore, the cardinality of the family $\mathcal{E}$ of all sets $A \subset Z$ such that $A=\bigcap \lambda$ for some countable subfamily $\lambda$ of the family $\mathcal{S} \cup \mathcal{G}$ also does not exceed $2^{\omega}$.

Let us show that for every $z \in(Z \backslash X)$ there exists $K \in \mathcal{E}$ such that $z \in K \subset$ $(Z \backslash X)$. Let $\mathcal{S}_{z}$ be the family of all $B \in \mathcal{S}$ such that $z$ is in the closure of $B \cap X$, and $\mathcal{G}_{z}$ the family of all $B \in \mathcal{G}$ such that $z \in B$. Consider the family

$$
\mathcal{P}_{z}=\left\{X \cap B: B \in\left(\mathcal{S}_{z} \cup \mathcal{G}_{z}\right)\right\} .
$$

The family $\mathcal{P}_{z}$ satisfies conditions imposed on $\mathcal{P}$ in Proposition 2.5. Therefore, there exists a countable subfamily $\eta$ of $\mathcal{S}_{z} \cup \mathcal{G}_{z}$ such that $\bigcap \eta \subset(Z \backslash X)$. Clearly, $z \in \bigcap \eta \in \mathcal{E}$.

It follows that there exists a subfamily $\mathcal{K}$ of $\mathcal{E}$ such that $Z \backslash X=\bigcup \mathcal{K}$. Since $|\mathcal{K}| \leq|\mathcal{E}| \leq 2^{\omega}$ and all elements of $\mathcal{K}$ are closed in $Z$, we conclude that $X$ is a $G_{2^{\omega}-\text { set. }}$

The following characterization of linearly Lindelöf spaces is well known.

Proposition 2.7. A space $X$ is linearly Lindelö if and only if every open covering of $X$ contains a subcovering $\gamma$ such that the cofinality of $\gamma$ is countable.

We denote by $l(X)$ the Lindelöf degree of $X$, that is, the smallest infinite cardinal number $\tau$ such that from every open covering of $X$ one can choose a subcovering, the cardinality of which does not exceed $\tau$.

Lemma 2.8. If $X$ is a linearly Lindelöf space such that the Lindelöf degree of $X$ does not exceed the first cardinal number $\tau$ which is greater than $2^{\omega}$, then $l(X) \leq 2^{\omega}$. 
Proof. This follows from Proposition 2.7.

The next assertion is also well known (and easily proved, see [5]).

Proposition 2.9. Let $X$ be a Tychonoff space and $\tau=l(X)$. Then for every Hausdorff compactification $b(X)$ of $X$ and every compact subset $K$ contained in $b(X) \backslash X$ there exists a $G_{\tau}$-set $P$ in $b(X)$ such that $K \subset P \subset b(X) \backslash X$.

The following lemma is obvious for Lindelöf spaces, while it is amazingly nontrivial for linearly Lindelöf spaces.

Lemma 2.10. Let $X$ be a linearly Lindelöf Tychonoff space and $Y$ a closed subspace of $X$ such that $X$ is first countable at each point of $Y,|Y| \leq 2^{\omega}$, and $l(X) \leq \tau$, where $\tau$ is the first cardinal number greater than $2^{\omega}$. Then $Y$ is a $G_{2^{\omega}}$-set in $X$.

Proof. Let $Z=b(X)$ be a Hausdorff compactification of $X$ and $H$ the closure of $Y$ in $Z$. Since $Y$ is first countable and $|Y| \leq 2^{\omega}$, the weight of $Y$ is not greater than $2^{\omega}$. It follows from Proposition 2.6 that $C h(Y) \leq 2^{\omega}$. Hence, we can fix a family $\eta=\left\{K_{\alpha}: \alpha<2^{\omega}\right\}$ of compact subspaces of $H$ such that $\bigcup \eta=H \backslash Y$.

By Lemma 2.8, $l(X) \leq 2^{\omega}$. Applying Proposition 2.9, we can fix a family $\gamma_{\alpha}$ of open sets in $Z$, for each $\alpha<2^{\omega}$, such that $\left|\gamma_{\alpha}\right| \leq 2^{\omega}$ and

$$
K_{\alpha} \subset \bigcap \gamma_{\alpha} \subset Z \backslash X
$$

For each $y \in Y$, we can also fix a countable base $\xi_{y}$ of $Z$ at $y$ (the space $Z$ is also first countable at each $y \in Y$, since $X$ is dense in $Z$ and $Z$ is regular).

Now let $\mathcal{U}$ be the family

$$
\left(\bigcup\left\{\gamma_{\alpha}: \alpha<2^{\omega}\right\}\right) \cup\left(\bigcup\left\{\xi_{y}: y \in Y\right\}\right) .
$$

Clearly, $|\mathcal{U}| \leq 2^{\omega}$, and given any $a$ in $H$ and $z \in(Z \backslash H)$, there is $V \in \mathcal{U}$ such that $a \in V$ and $z \notin V$. From compactness of $H$ it follows that the family $\mu$ of all open neighbourhoods $W$ of $H$ in $Z$ such that $W$ is the union of a finite subcollection of

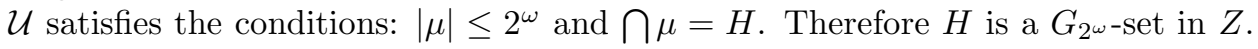
Then $Y$ is a $G_{2}$-set in $X$.

A space $X$ is $\omega_{1}$-Lindelöf, if every open covering $\gamma$ of $X$ such that $|\gamma| \leq \omega_{1}$ contains a countable subcovering. Every linearly Lindelöf space is $\omega_{1}$-Lindelöf.

Lemma 2.11. Let $X$ be a first countable $\omega_{1}$-Lindelöf Hausdorff space satisfying the next condition:

c) every closed subset $A$ of $X$ such that $|A| \leq 2^{\omega}$ is a $G_{2^{\omega}-\text { set }}$ in $X$.

Then $|X| \leq 2^{\omega}$.

Proof. This is a standard saturation proof (see [3], [4], [6]). Let $\mathcal{P}$ be the family of all closed subsets $A$ of $X$ such that $|A| \leq 2^{\omega}$. For each $A \in \mathcal{P}$ we fix a family $\eta(A)$ of open sets in $X$ such that $|\eta(A)| \leq 2^{\omega}$ and $\bigcap \eta(A)=A$. For every countable family $\gamma$ of open sets in $X$ such that $X \backslash \bigcup \gamma$ is not empty, we fix a point $c_{\gamma}$ in $X \backslash \bigcup \gamma$. Now we are going to define by transfinite recursion an $\omega_{1}$-long sequence $\left\{A_{\alpha}: \alpha<\omega_{1}\right\}$ of elements of $\mathcal{P}$.

Put $A_{0}=\emptyset$. Let us assume that $A_{\beta} \in \mathcal{P}$ is already defined for every $\beta<\alpha$, for some ordinal $\alpha<\omega_{1}$. We will now define the set $A_{\alpha} \in \mathcal{P}$. Put $\mathcal{U}_{\alpha}=\bigcup\left\{\eta\left(A_{\beta}\right)\right.$ : $\beta<\alpha\}$, and let $\mathcal{W}$ be the family of all countable subfamilies $\gamma$ of the family $\mathcal{U}$ such that $X \backslash \bigcup \gamma \neq \emptyset$. Put $C_{\alpha}=\left\{c_{\gamma}: \gamma \in \mathcal{W}\right\}$ and $H_{\alpha}=\left(\bigcup\left\{A_{\beta}: \beta<\alpha\right\}\right) \cup C_{\alpha}$. Clearly, $|\mathcal{U}| \leq 2^{\omega},|\mathcal{W}| \leq 2^{\omega}$, and $\left|H_{\alpha}\right| \leq 2^{\omega}$. Let $A_{\alpha}$ be the closure of the set $H_{\alpha}$ 
in $X$. Then $\left|A_{\alpha}\right| \leq 2^{\omega}$, since $X$ is first countable and Hausdorff. Hence, $A_{\alpha} \in \mathcal{P}$. The definition of the sets $A_{\alpha}$ for each $\alpha<\omega_{1}$ is complete.

It is clear from the construction that if $\beta<\alpha$, then $A_{\beta} \subset A_{\alpha}$. Since the tightness of $X$ is countable and each $A_{\alpha}$ is closed in $X$, it follows that the set $L=\bigcup\left\{A_{\alpha}: \alpha<\omega_{1}\right\}$ is closed in $X$. Therefore, the subspace $L$ of $X$ is $\omega_{1}$-Lindelöf. Also $|L| \leq 2^{\omega}$.

Let us show that $L=X$. Assume the contrary, and fix $b \in X \backslash L$. Then, for each $\alpha<\omega_{1}$, we can choose $U_{\alpha} \in \eta\left(A_{\alpha}\right)$ such that $b$ is not in $U_{\alpha}$. Also, $A_{\alpha} \subset U_{\alpha}$. Therefore, the family $\left\{U_{\alpha}: \alpha<\omega_{1}\right\}$ is an open covering of $L$. This covering contains a countable subfamily $\gamma$ such that $L \subset \bigcup \gamma$, since $L$ is $\omega_{1}$-Lindelöf. Then $b$ is not in $\bigcup \gamma$; therefore, a point $c_{\gamma}$ is defined such that $c_{\gamma} \in X \backslash \bigcup \gamma \subset X \backslash L$. On the other hand, since $\omega_{1}$ is not countably cofinal and $\gamma$ is countable, there is $\alpha^{*}<\omega_{1}$ such that $\gamma \subset \mathcal{U}_{\alpha^{*}}$ and $\gamma \in \mathcal{W}_{\alpha^{*}}$. Then $c_{\gamma} \in C_{\alpha^{*}} \subset H_{\alpha^{*}} \subset A_{\alpha^{*}} \subset L$, a contradiction. The proof of Lemma 2.11 is complete.

Now we have almost all of the auxiliary results we need to prove the two main theorems in the next section.

\section{§3. The Main theOrems, SOME COROllaries, AND OPEN QUeSTIONS}

Theorem 3.1. The cardinality of every linearly Lindelöf first countable Tychonoff space does not exceed $2^{\omega}$.

Proof. Assume the contrary. Then, by Lemma 2.1, there exists a linearly Lindelöf first countable Tychonoff space $X$ such that $|X|$ is the first cardinal number $\tau$ which is greater than $2^{\omega}$. Then $l(X) \leq \tau$ and, by Lemma 2.10, every closed subset $A$ of $X$

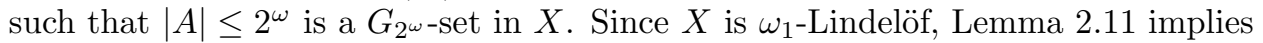
that $|X| \leq 2^{\omega}<\tau$, a contradiction.

Proposition 3.2. Let $X$ be a Tychonoff space such that the closure of every countable discrete subspace of $X$ is Lindelöf and all free sequences in $X$ are countable. Then $X$ is Lindelöf.

Proof. Let $Z=b(X)$ be any Hausdorff compactification of $X$. To prove that $X$ is Lindelöf, it suffices to show that each point of $Z \backslash X$ is $G_{\omega}$-separated from $X$ (see [5]).

Assume the contrary, and fix a point $z \in Z \backslash X$ which is not $G_{\omega}$-separated from $X$. Since all free sequences in the space $X$ are countable, from Lemma 2.2 it follows that there is a countable discrete (in itself) subset $B$ of $X$ such that $z$ is not $G_{\omega}$-separated from the closure $Y$ of $B$ in $X$. On the other hand, the space $Y$ is Lindelöf, by the assumptions in the theorem. Therefore, $z$ is $G_{\omega}$-separated from $Y$ (in the closure of $Y$ in $Z$ and, therefore, in the larger space $Z$ as well). This contradiction completed the proof of Proposition 3.2.

Theorem 3.3. Let $X$ be an $\omega_{1}$-Lindelöf Tychonoff space of countable tightness such that the closure of every countable discrete subspace of $X$ is Lindelöf. Then $X$ is Lindelöf.

Proof. In virtue of Proposition 3.2, it is enough to refer to the following well known and easy to prove result: in an $\omega_{1}$-Lindelöf space of countable tightness all free sequences are countable (see [2], [3]).

In particular, Proposition 3.2 and Theorem 3.3 apply to linearly Lindelöf spaces. 
Corollary 3.4. Under $(C H)$, every $\omega_{1}$-Lindelöf Tychonoff space $X$ of countable tightness is Lindelöf.

Proof. Indeed, under $(\mathrm{CH})$, the weight of the closure of any countable subspace of $X$ does not exceed $\omega_{1}$, and every $\omega_{1}$-Lindelöf space of the weight not greater than $\omega_{1}$ is obviously Lindelöf. Therefore, under $(\mathrm{CH})$, the closure in $X$ of any countable discrete subspace of $X$ is Lindelöf. It remains to apply Theorem 3.3.

Recall that a space $X$ is said to be strongly discretely Lindelöf, if the closure of every discrete subspace of $X$ is Lindelöf [4]. It is still an open question, whether every strongly discretely Lindelöf Tychonoff space is Lindelöf [4]. On the other hand, every strongly discretely Lindelöf space is linearly Lindelöf [4]. Therefore, the next result is a particular case of Theorem 3.3.

Corollary 3.5. Every strongly discretely Lindelöf Tychonoff space of countable tightness is Lindelöf.

Modifying slightly the proof of Theorem 3.3, we obtain the following generalization of Corollary 3.5:

Theorem 3.6. Every strongly discretely Lindelöf Tychonoff space such that the tightness of $X$ is less than $\aleph_{\omega}$, is Lindelöf.

Proof. Assume the contrary. Then we can fix a Hausdorff compactification $Z=$ $b(X)$ of $X$ and a point $b \in Z \backslash X$ such that $b$ is not $G_{\omega}$-separated from $X$. Let $\tau$ be the first cardinal number which is greater than the tightness of $X$. Then $\tau<\aleph_{\omega}$. Since $X$ is linearly Lindelöf and $b$ is not $\omega$-separated from $X$, it follows that $b$ is not $\tau$-separated from $X$.

Now note that a $\tau$-version of Lemma 2.2 is proved by the same argument as Lemma 2.2. In fact, what we really need is a strengthened $\tau$-version of Lemma 2.3, which immediately follows from the $\tau$-version of Lemma 2.2. Here it is:

Lemma 3.7. Let $Y$ be a subspace of $X, x \in X$, and $\tau$ a cardinal number such that $F(Y) \leq \tau$. Then either

a) There is a free sequence $A$ in $Y$ such that $|A| \leq \tau$ and $x$ is not $G_{\tau}$-separated from the closure of $A$ in $Y$, or

b) $x$ is $G_{\tau}$-separated from $Y$.

This Lemma completes the proof of Theorem 3.6.

Note another result which also easily follows from Lemma 3.7.

Proposition 3.8. Let $X$ be a Tychonoff space and $\tau$ a cardinal number such that $F(X) \leq \tau$ and the Lindelof degree of the closure of any free sequence $A$ in $X$ does not exceed $\tau$. Then the Lindelöf degree of $X$ is not greater than $\tau$.

A space $X$ is called $\omega$-monolithic [3], if the closure of any countable subset of $X$ has a countable network. From Proposition 3.2 and Theorem 3.3 we immediately get the following corollaries:

Corollary 3.9. Every $\omega$-monolithic Tychonoff space $X$ such that all free sequences in $X$ are countable, is Lindelöf.

Corollary 3.10. Every $\omega$-monolithic linearly Lindelöf Tychonoff space of countable tightness is Lindelöf. 
Question 3. Is it true in ZFC that every first countable linearly Lindelöf Tychonoff space is Lindelöf? Can one drop $(\mathrm{CH})$ in the formulation of Corollary 3.4? Is every hereditarily separable linearly Lindelöf Tychonoff (or just regular) space Lindelöf?

Note, that every linearly Lindelöf space with a point-countable base is Lindelöf. In fact, every metaLindelöf linearly Lindelöf space is Lindelöf, since in a linearly Lindelöf space every closed discrete subspace is countable, and an easy standard "stars of the points" argument shows that every metaLindelöf space with this property is Lindelöf (see [5]).

Question 4. Is every Hewitt-complete linearly Lindelöf Tychonoff space Lindelöf?

Question 5. Is every locally compact linearly Lindelöf Hausdorff space Lindelöf?

Question 6. Is every locally metrizable linearly Lindelöf Tychonoff space Lindelöf?

Question 7. Is there a consistent example of a pseudocompact locally compact locally metrizable linearly Lindelöf non-compact space?

Question 8. Is there a consistent example of a topology on the real line which is stronger than the usual topology and is linearly Lindelöf, locally compact, normal, and not metrizable (and, therefore, not Lindelöf)?

\section{§4. An example And a Related Result}

We present here an example of a linearly Lindelöf Tychonoff non-Lindelöf space which seems to be simpler than Mischenko's example. This example was discovered independently by G. Gruenhage and R. Buzyakova.

Example 4.1. Let $D$ be the standard discrete two-point set with elements 0 and 1 , and $\tau=\aleph_{\omega}$, that is, $\tau$ is the first uncountable cardinal number cofinal to $\omega$. Fix a set $A$ of cardinality $\tau$, and consider the product space $D^{A}$, with the usual product topology. For a point $x \in D^{A}$ we denote by $A_{x}$ the set of all $a \in A$ such that the corresponding coordinate $x_{a}$ of $x$ is 1 . Let $X$ be a subspace of $D^{A}$ consisting of all points $x \in D^{A}$ such that the cardinality of $A_{x}$ is smaller than $\tau$.

Clearly, $X$ is a Tychonoff space. Let us show first that $X$ is not Lindelöf. Indeed, $X$ is dense in $D^{A}$ but $X$ does not coincide with $D^{A}$. Therefore, $X$ is not closed in $D^{A}$, which implies that $X$ is not compact. On the other hand, the $\Sigma$-product subspace of $D^{A}$, consisting of all $x \in D^{A}$ such that the set $A_{x}$ is countable, is a countably compact subspace of $X$, dense in $D^{A}$ and, therefore, dense in $X$ (see [5]). It follows that $X$ is pseudocompact. We now conclude that $X$ is not Lindelöf, since every pseudocompact Lindelöf space is compact.

Now let us prove that $X$ is linearly Lindelöf. Take any uncountable subset $B$ of $X$ such that $|B|$ is a regular cardinal. Clearly, the weight of $X$ is not greater than $\tau$, since $\tau$ is the weight of $D^{A}$. Therefore, the Lindelöf degree of $X$ is not greater than $\tau$, which implies that if $|B|>\tau$, then $B$ has a point of complete accumulation in $X$.

Since $\tau$ is not regular, it remains to consider the case when $|B|<\tau$. We can fix a cardinal $\tau_{n}<\tau$, for each $n \in \omega$, in such a way that $\tau$ is the supremum of $\left\{\tau_{n}\right.$ : $n \in \omega\}$. Let $X_{n}$ be the set of all $x \in X$ such that $\left|A_{x}\right| \leq \tau_{n}$, for $n \in \omega$. Obviously $X=\bigcup\left\{X_{n}: n \in \omega\right\}$. Put $B_{n}=B \cap X_{n}$, for $n \in \omega$. Then $B=\bigcup\left\{B_{n}: n \in \omega\right\}$ and, for some $k \in \omega,\left|B_{k}\right|=|B|$, since $|B|$ is regular and uncountable. Therefore, 
it would suffice to show that there is a point of complete accumulation for $B_{k}$ in $X$. We will prove more: that the closure of $B_{k}$ in $X$ is compact.

There is $m \in \omega$ such that $\tau_{k} \leq \tau_{m}$ and $\left|B_{k}\right|=|B|<\tau_{m}$. Put $A(m)=\bigcup\left\{A_{x}: x \in\right.$ $B_{k}$ \} and let $K$ be the set of all points $x \in X$ such that $x_{a}=0$ for each $a \in A \backslash A(m)$. It is easy to see from these definitions that $K$ is a compact subspace of $X$ and that $B_{k}$ is a subset of $K$. Therefore, $B_{k}$ has a point of complete accumulation in $K$ and in $X$. Thus, $X$ is linearly Lindelöf.

Note two more properties of the space $X$ : it is a topological group (and therefore is topologically homogeneous) and the Souslin number of $X$ is countable (since $X$ is dense in $D^{A}$ ).

It is interesting to compare this example with the following special version of Theorem 3.6.

Theorem 4.2. Assume $(G C H)$, and let $X$ be a linearly Lindelöf Tychonoff space such that $t(X)<\aleph_{\omega}$. Then $X$ is Lindelöf.

Proof. We argue as in the proof of Theorem 3.6, taking into account that for every discrete subset $A$ of $X$ such that $|A|<\aleph_{\omega}$, the closure of $A$ in $X$ is Lindelöf, which follows from $(G C H)$ (this clearly suffices, we do not really need to know that $X$ is discretely Lindelöf).

Observe, that if we could drop $(G C H)$ in the theorem above, it would become the best possible result of this kind, since in Example $4.1 X$ is a linearly Lindelöf non-Lindelöf space the tightness of which is exactly $\aleph_{\omega}$.

Question 9. Does Theorem 4.2 remain valid if we drop $(G C H)$ from its formulation?

Question 10. Is every strongly discretely Lindelöf Tychonoff space Lindelöf?

It is clear how to define the linear Lindelöf degree $l l(X)$ of a space $X$. Once this is done, we could easily prove the obvious $\tau$-versions of Theorems $3.1,3.3$, and 4.1, for any cardinal number $\tau$, virtually by the same argument.

\section{NOTE ADDED IN PROOF}

Recently Arhangel'skii and Buzyakova proved that consistently the answer to Question 4 is "no", the answer to Question 6 is "yes", and the answers to Question 7 and Question 8 are negative.

\section{REFERENCES}

[1] Alexandroff P.S. and P.S. Urysohn, Memoire sur les espaces topologiques compacts, Nederl. Akad. Wetensch. Proc. Ser. A, 14 (1929), 1-96.

[2] Arhangel'skii A.V., On the cardinality of bicompacta satisfying the first axiom of countability, Soviet Math. Dokl. 10 (1969), 951-955.

[3] Arhangel'skii A.V., Structure and classification of topological spaces and cardinal invariants, Russian Math. Surveys 33 (1978), 33-96.

[4] Arhangel'skii A.V., A generic theorem in the theory of cardinal invariants of topological spaces, Comment. Math. Univ. Carolinae 36:2 (1995), 303-325. MR 97b:54005

[5] Engelking R., General Topology, Sigma Series in Pure Mathematics, 6, Heldermann, Berlin, revised ed., 1989. MR 91c:54001

[6] Hodel R.E., Cardinal Functions, 1, in: Handbook of Set-theoretic Topology, Editors: K. Kunen and J.E. Vaughan, Chapter 1, 1-62, North-Holland, Amsterdam, 1984. MR 86j:54007 
[7] Mischenko A.S., Finally compact spaces, Soviet Math. Dokl. 145 (1962), 1199-1202.

[8] Rudin M.E., Some Conjectures, in: J. van Mill and G.M Reed, Editors, Open Problems in Topology (1990), 184-193, North-Holland, Amsterdam. CMP 91:03

(A. V. Arhangel'skii) July-December: Department of Mathematics, Moscow State UniVERSITY, Moscow 119899, Russia

E-mail address: arhangel@nw.math.msu.su

January-June: Department of Mathematics, Ohio State University, Athens, Ohio 45701

E-mail address: arhangel@bing.math.ohiou.edu

(R. Z. Buzyakova) Chair of General Topology and Geometry, Mech.-Math. Faculty, Moscow State University, Moscow 119899, Russia

E-mail address: raushan@shade.msu.ru 